\title{
Resistencia y deformación de concretos elaborados con agregados disponibles en el Valle de México
}

\author{
O. Hernández-Castañeda y A.L. Fuentes-González \\ División de Estudios de Posgrado, Facultad de Ingeniería, UNAM e Instituto de Ingeniería, UNAM. \\ E-mails: ohcastaneda@correo.unam.mxyafug@pumas.iingen.unam.mx
}

(recibido: ènero de 2002; aceptado: julio de 2002)

\section{Resumen}

Esta investigación tiene el objetivo de analizar el comportamiento y respuesta de concretos fabricados con distintos tipos de agregados disponibles en el Valle de México, con el fin de determinar su influencia en las propiedades del concreto y su mejor aplicación en la industria de la construcción.

Se analiza el desarrollo de la resistencia a compresión y la deformabilidad a largo plazo (flujo plástico) de los concretos investigados. Los datos experimentales muestran la importancia y el efecto del agregado en la resistencia y deformación del concreto.

Adicionalmente, se proponen ecuaciones de flujo plástico para los concretos investigados, indispensables en el diseño estructural. La consideración del flujo plástico o deformación diferida del concreto en el diseño estructural, permite aumentar la vida útil y minimizar el costo de mantenimiento de aquellas estructuras elaboradas con este noble material.

Descriptores: concreto, agregado, resistencia a compresión, deformación, flujo plástico.

\section{Alstract}

This research project has the main objective of analyzing the behavior and response of concretes made from different types of disposable aggregates available in the Valley of M Ievico. Our purpose is to determine their influence on the properties of concrete and their most sutulle applications in the construction industry.

We analyze the development of compressive strength and creep of the different concretes in question. The experimental results show the importance and effect of a given aggregate on the strength and creep of concrete.

Additionally, creep equations for these concretes are proposed, as they are indispensable on structural design. Taking into account creep factors in the structural design phase can help to increase the useful life and minimize the maintenance costs of those structures built with concrete.

Keywords: concrete, aggregate, compressive strength, deformation, creep. 


\section{Introducción}

Para brindar una seguridad estructural óptima es primordial realizar un adecuado diseño estructural, emplear materiales estructurales que cumplan con las especificaciones, así como una adecuada práctica constructiva; sólo con la interacción estrecha de tales elementos es posible brindar una seguridad estructural real.

El concreto estructural es uno de los materiales más empleados en la construcción, por ello, los ingenieros civiles y constructores requerimos conocer su tecnología.

Tradicionalmente, el criterio para la utilización de un concreto ha sido su resistencia a compresión, y es hasta los últimos años, cuando ha surgido un interés por la durabilidad del mismo.

Sin embargo, la deformación del concreto es otro factor importante a considerar en el diseño estructural, para incrementar la vida útil de las estructuras y disminuir su costo de mantenimiento a largo plazo. Entre las deformaciones y cambios volumétricos que presenta el concreto, el flujo plástico - creep, por su nombre en inglés- y la contracción por secado, ocupan un lugar primordial, por lo que en este trabajo se desarrollan ambos aspectos.

Una variable interesante en este estudio es el agregado. Anteriormente se consideraba el papel del agregado en el concreto como secundario; sin embargo, varias investigaciones en diferentes partes del mundo han indicado que esto no es así, y que la función del agregado en el concreto tiene más implicaciones de las que se pensaba inicialmente. Por lo anterior, el Instituto de Ingeniería de la UNAM, desde hace algunos años ha analizado varios concretos estructurales con los agregados disponibles en el Valle de México. En este trabajo se hace una recopilación de los datos más representativos, donde se confrontan los resultados experimentales de concretos fabricados con diferentes agregados disponibles en el Valle de México, para ello se analizó la resistencia y la deformabilidad, características importantes para la evaluación de un concreto.

\section{Materiales}

Para conocer el efecto en el concreto de algunos agregados disponibles en el Valle de México, se elaboraron concretos con varias combinaciones de agregados. Los concretos fabricados comprenden tres tipos de agregados distintos, y por supuesto, una dosificación diferente. Así, las variables a analizar son el tipo de agregado y el consumo de cemento ${ }^{1}$. En este artículo se exponen las propiedades de resistencia a compresión y flujo plástico, y en la segunda parte se discutirá la contracción por secado.

Se analizaron 12 mezclas de concreto. Las mezclas se dosificaron con el criterio ACI 211.1-91 (1997), su proporcionamiento y los materiales empleados se muestran en la tabla 1.

\section{Cemento Portland}

Para la investigación se emplearon dos tipos de cementos: cemento Portland ordinario y cemento Portland puzolánico, ambos de producción nacional. Se seleccionaron estos tipos de cemento por ser los más empleados en la industria mexicana de la construcción.

\section{Agregados}

Antes de los sismos de 1985 se empleaban principalmente agregados pétreos andesíticos para la fabricación de concreto hidráulico en la Ciudad de México, debido a su fácil disponibilidad en la región. Las fallas de estructuras en los sismos de 1985 fueron originadas, entre otras causas, por el contacto entre estructuras con gralean, concretos fabricados con materiales andesíticos. En estudios anteriores (Mendoza, et al., 1993) se ha demostrado que el comportamiento de dichos concretos es satisfactorio en el ámbito de la resistencia mecánica, pero no en el ámbito del flujo plástico, ni en su módulo de elasticidad; comparado con otros agregados el material andesítico presenta un flujo plástico y una contracción por secado, del orden del doble de otros materiales pétreos. Aunado a lo anterior, su

\footnotetext{
1 Es preferible considerar una a la vez, las propiedades significativas del concreto, pero en enfoques experimentales esto es difícilmente posible, debido fundamentalmente a que ciertas variables son covariables. Un ejemplo, es que al variar el consumo de cemento cambiamos también la relación agua/cemento y se puede afectar su trabajabilidad. Lo anterior dificulta el análisis del concreto.
} 
DOI: http://dx.doi.org/10.22201/fi.25940732e.2003.04n1.001

O. Hernández-Castañeda y A.L. Fuentes-González

Tabla 1. Características analizadas de las mezclas de concreto

\begin{tabular}{|c|c|c|c|c|c|c|}
\hline \multirow{2}{*}{ DESIGNACIÓN } & \multirow{2}{*}{\multicolumn{2}{|c|}{ TIPO DE CEMENTO }} & \multicolumn{3}{|c|}{ ORIGEN GEOLÓGICO DEL AGREGADO } & \multirow{2}{*}{$\begin{array}{l}\text { TAM } \\
\text { MAX }\end{array}$} \\
\hline & & & \multicolumn{2}{|c|}{ Grava } & Arena & \\
\hline A1 & \multicolumn{2}{|c|}{ Portland Puzolánico } & \multicolumn{2}{|c|}{ Caliza } & Caliza & $1 "$ \\
\hline A2 & \multicolumn{2}{|c|}{ Portland Puzolánico } & \multicolumn{2}{|c|}{ Caliza } & Caliza & $1^{\prime \prime}$ \\
\hline $\mathrm{A} 3$ & \multicolumn{2}{|c|}{ Portland Puzolánico } & \multicolumn{2}{|c|}{ Caliza } & Caliza & $1^{\prime \prime}$ \\
\hline A4 & \multicolumn{2}{|c|}{ Portland Puzolánico } & \multicolumn{2}{|c|}{ Caliza } & Caliza & $11 / 2 "$ \\
\hline A5 & \multicolumn{2}{|c|}{ Portland Puzolánico } & \multicolumn{2}{|c|}{ Caliza } & Caliza & $1 \frac{1 / 2}{\prime \prime}$ \\
\hline A6 & \multicolumn{2}{|c|}{ Portland Puzolánico } & \multicolumn{2}{|c|}{ Caliza } & Caliza & $1 \frac{1 / 2}{\prime \prime}$ \\
\hline B7 & \multicolumn{2}{|c|}{ Portland, Tipo I } & \multicolumn{2}{|c|}{ Caliza } & Andesita & $3 / 8 "$ \\
\hline B8 & \multicolumn{2}{|c|}{ Portland, Tipo I } & \multicolumn{2}{|c|}{ Caliza } & Andesita & $3 / 8 "$ \\
\hline B9 & \multicolumn{2}{|c|}{ Portland, Tipo I } & \multicolumn{2}{|c|}{ Caliza } & Andesita & $3 / 8 "$ \\
\hline C10 & \multicolumn{2}{|c|}{ Portland, Tipo I } & \multicolumn{2}{|c|}{ Basalto } & Arena de río & $3 / 4:$ \\
\hline C11 & \multicolumn{2}{|c|}{ Portland, Tipo I } & \multicolumn{2}{|c|}{ Basalto } & Arena de río & $3 / 4 a$ \\
\hline $\mathrm{C} 12$ & \multicolumn{2}{|c|}{ Portland, Tipo I } & \multicolumn{2}{|c|}{ Basalto } & Arena de río & $3 / 4 "$ \\
\hline \multirow{4}{*}{ DESIGNACIÓN } & & DOSIF & ÓN & & RELACIC & ICAS \\
\hline & Consumo de & Grava & Arena & Agua & Agua/ & Agregado/ \\
\hline & cemento & & & & Cemento & Cemento \\
\hline & {$\left[\mathrm{kg} / \mathrm{m}^{3}\right]$} & {$\left[\mathrm{kg} / \mathrm{m}^{3}\right]$} & {$\left[\mathrm{kg} / \mathrm{m}^{3}\right]$} & {$\left[\mathrm{kg} / \mathrm{m}^{3}\right]$} & $\mathrm{A} / \mathrm{C}$ & $\mathrm{AG} / \mathrm{C}$ \\
\hline A1 & 319 & 960 & 821 & 204 & 0.64 & 5.58 \\
\hline A2 & 381 & 1008 & 729 & 208 & 0.55 & 4.56 \\
\hline $\mathrm{A} 3$ & 491 & 993 & 598 & 226 & 0.46 & 3.24 \\
\hline A4 & 454 & 836 & 812 & 213 & 0.47 & 3.63 \\
\hline A5 & 364 & 842 & 888 & 207 & 0.57 & 4.75 \\
\hline A6 & 317 & 842 & 927 & 201 & 0.63 & 5.58 \\
\hline B7 & 350 & 1026 & 778 & 169 & 0.48 & 5.15 \\
\hline B8 & 400 & 1002 & 758 & 170 & 0.43 & 4.40 \\
\hline B9 & 493 & 948 & 718 & 166 & 0.34 & 3.38 \\
\hline $\mathrm{C} 10$ & 400 & 952 & 753 & 171 & 0.43 & 4.26 \\
\hline C11 & 450 & 924 & 731 & 172 & 0.38 & 3.68 \\
\hline $\mathrm{C} 12$ & 500 & 908 & 718 & 150 & 0.30 & 3.25 \\
\hline MÍNIMO & 317 & 836 & 598 & 150 & 0.30 & 3.24 \\
\hline MÁXIMO & 500 & 1026 & 927 & 226 & 0.64 & 5.58 \\
\hline
\end{tabular}

módulo de elasticidad es del orden de la mitad comparado con otros agregados pétreos (Mendoza et al., 1995).

El sismo de 1985 en la Ciudad de México demostró la deficiencia en los materiales empleados, fue entonces cuando se pensó en mejorar el comportamiento de los agregados con los que se elabora el concreto. Por lo anterior, se estudió y posteriormente se reglamentó el uso de un concreto más rígido (menos deformable); se propuso fabricar el concreto con un agregado grueso de origen basáltico o calizo (más denso que el andesítico); sin embargo, el agregado fino (arena) de origen andesítico se siguió utilizando.

Este último concreto alcanza razonablemente las condiciones deseables para concreto estructural, pero hay circunstancias donde es conveniente un concreto más denso y más rígido. Por ejemplo, en las cimentaciones total y 
parcialmente compensadas, un concreto más denso significa menos volumen de concreto y menos volumen de excavación, entre otros hechos benéficos. Para lograrlo, se propone usar material calizo $\left(2.60 \mathrm{t} / \mathrm{m}^{3}\right)$ en la arena, en lugar de uno de andesita $\left(2.40 \mathrm{t} / \mathrm{m}^{3}\right)$.

Por situaciones geológicas, la arena andesita es la de mejor calidad en el Valle de México; transportar una arena de otra región resulta costoso; sin embargo, si los materiales cercanos a la región no satisfacen los requerimientos de calidad, conviene transportar los agregados de regiones lejanas, esto para asegurar la calidad del concreto. Una solución alterna es la trituración de rocas de la región, hasta obtener la granulometría óptima de la arena.

\section{Caliza}

La caliza es una roca sedimentaria que por sus características se le considera un excelente material para la construcción. El primer lote de concretos fabricado contiene grava y arena de origen calizo, se trituraron rocas calizas hasta obtener la granulometría de la arena adecuada.

El uso de arenas trituradas tiene consecuencias en el concreto fresco, ya que las mezclas tienden a ser menos trabajables, proclives a la segregación $y$ al sangrado; por ello, resulta útil y de interés estudiar mezclas de concreto elaboradas con agregados triturados calizos, tanto gruesos como finos y conocer sus propiedades en estado fresco y endurecido, así como su comportamiento a través del tiempo.

\section{Andesita}

La andesita es una roca ígnea extrusiva, tipo lava, y se localiza en gran parte de la cuenca del Valle de México. Se encuentra en la Sierra Nevada, el Ajusco, las Sierras de las Cruces y Monte Alto, y en gran parte del abanico de origen fluvio - glacial que cubre las faldas de las Sierras del Sur de la Ciudad de México, asimismo, en el Estado de México: al oriente de Texcoco, en los Montes Ocuilan, en Maninalco y en Malitenango. En el estado de Morelos se localiza en las "Lomas" de
Cuernavaca (Rico y Del Castillo, 2000). Para tener un parámetro de comparación con los concretos elaborados con ambos agregados calizos, se elaboraron concretos con agregados pétreos, tales como grava caliza y arena andesita de procedencia local; estudios previos demostraron que estos materiales lograban un concreto estructural satisfactorio, con las salvedades marcadas anteriormente.

\section{Basalto}

El basalto es una roca ígnea extrusiva, tipo lava, muy abundante en la República, principalmente en el Valle de México y sus alrededores (Rico y Del Castillo, 2000). El Departamento del Distrito Federal encontró un banco de basalto, el cual por resultar un material viable para producir concreto podría abastecer la demanda de agregado del Valle de México para los próximos 20 años. Su desempeño para la elaboración de concreto se conoce poco en México; se trata de un material poco estudiado y poco empleado para obras importantes de ingeniería civil. De ahí su importancia de investigar su aplicación y desempeño en concretos estructurales.

Ante la necesidad de encontrar materiales sustitutos de los ya escasos y empleados tradicionalmente, el basalto surge como posible material alterno para producir concreto, por lo que su análisis para determinar su viabilidad en concretos estructurales es primordial.

\section{Arena de río}

La arena de río puede ser a provechada cuando se construyen obras que están próximas a un río o una corriente de agua, disminuyendo el costo; tal es el caso de presas que se ubican en cuencas que cuentan con grandes cantidades de arena de río. La Comisión Nacional del Agua (CNA) plantea usar dicho material para canales de descarga, donde es necesario un recubrimiento de concreto resistente al flujo del agua con acabado liso. El empleo de arenas de río para elaborar concreto hidráulico surge como una nueva necesidad, por lo que carece de estudios anteriores, de ahí su interés por 
analizar la respuesta de un concreto fabricado con éste agregado. Normalmente se utilizan arenas provenientes de un banco que garantiza la calidad de los materiales; a diferencia de lo anterior, se propone analizar un material que se encuentra in situ, de fácil disponibilidad, pero sin antecedentes en su empleo.

Las propiedades del concreto con arena de río deben conocerse para poder determinar su viabilidad con fines estructurales, y en especial, para estructuras hidráulicas, como canales de descarga, así como para conocer el proporcionamiento de materiales más adecuado. Por lo anterior, el tercer lote de concretos investigados se realizaron con arena de río. Ante dichas circunstancias, el Instituto de Ingeniería se ha dedicado a este estudio, por ello se ha considerado arena de río para este artículo.

Por la diversidad de materiales existentes y provenientes de bancos del Valle de México, es conveniente identificar la influencia particular de aquellos que se encuentren en mayor volumen, y que sus condiciones geológicas les permitan ser empleados como agregado para producir concreto. Sin olvidar aquellos bancos con materiales alternos y poco conocidos que se pueden ocupar en el futuro para la fabricación de concreto.

En el presente estudio se han incluido los agregados más representativos y más utilizados para la construcción en el Valle de México, así como aquellos que requieren un análisis más amplio para su aplicación.

Se estudiaron concretos elaborados con grava y arena caliza (primer lote), grava caliza y arena andesita (segundo lote), y con grava de basalto y arena de río (tercer y último lote). Con la determinación y comparación de las propiedades del concreto que cada combinación de agregado produce, se determinó la influencia de cada uno y se identificaron las circunstancias en que puedan convenir.

\section{Propiedades del concreto en estado fresco}

Los valores promedio obtenidos para el concreto fresco son los indicados en la tabla 2.

\section{Metodología de análisis}

Debido a la diversidad de mezclas y a la cantidad de variables a analizar, se eligió como criterio, el consumo de cemento para comparar los concretos elaborados. Los concretos fabricados se dividieron en cinco intervalos, de acuerdo al consumo de cemento que presentan las mezclas; cada uno tiene un $5.0 \%$ de variación entre la media y los valores extremos del intervalo en consumo de cemento, lo que facilita la comparación y no afecta significativamente la validez de la comparación (Tabla 3).

\section{Resistencia a compresión}

Con el propósito de evaluar la influencia e importancia del agregado y del consumo de cemento en la resistencia mecánica del concreto endurecido, se determinó la resistencia a compresión para varias edades. Las propiedades en estado endurecido de los concretos investigados, se presentan en la tabla 4.

Se puede visualizar en las figuras 1 a 4 (todas las figuras se encuentran al final del artículo), el desarrollo de la resistencia a compresión con los agregados analizados, ahí se aprecia que a mayor consumo de cemento mayor resistencia a la compresión.

La estimación del desarrollo de resistencia a compresión, se realizó con el método propuesto por el Comité ACI 209R-92 (ratificado en 1997) para las condiciones de curado y almacenado de especímenes, y se confronta con los resultados experimentales obtenidos.

En las figuras 5 a 9 se comparan los desarrollos de resistencias a compresión de concretos con un consumo de cemento similar o igual, elaborados con diferentes agregados, donde se muestra la relevante influencia del agregado en la resistencia a compresión, ya que para un mismo o similar consumo de cemento, el concreto presenta diferencias sustanciales en la resistencia a compresión, causadas principalmente por la combinación de los agregados empleados. La mayor diferencia se presenta entre un concreto con la grava de basalto y arena de río y otro con grava caliza y arena caliza sin lavar, que en promedio, es próxima al $89 \%$. La segunda 
Tabla 2. Propiedades promedio en estado fresco según el tipo de agregado

\begin{tabular}{lccc}
\hline \multicolumn{1}{c}{ TIPO DE AGREGADO } & Revenimiento $(\mathrm{cm})$ & Aire incluido [\%] & $\mathrm{PVF}^{*}\left[\mathrm{~kg} / \mathrm{m}^{3}\right]$ \\
\hline I. Grava caliza y arena caliza sin lavar & 7.1 & 1.4 & 2328.7 \\
II. Grava caliza y arena caliza lavada & 8.8 & 2.2 & 2311.2 \\
III. Grava caliza y arena andesita & 13.3 & 2.0 & 2336.7 \\
IV. Grava basalto y arena de río & 1.4 & 2.1 & 2300.2 \\
\hline
\end{tabular}

*PVF. Peso volumétrico fresco

Tabla 3. Intervalos de comparación con base en el consumo de cemento

\begin{tabular}{cccc}
\hline & Intervalo & Media & Mezclas \\
\hline 1 & $310<\mathrm{CC}<326$ & 318 & $\mathrm{~A} 1, \mathrm{~A} 6$ \\
2 & $348<\mathrm{CC}<366$ & 366 & $\mathrm{~A} 5, \mathrm{~B} 7$ \\
3 & $380<\mathrm{CC}<401$ & 391 & $\mathrm{~A} 2, \mathrm{~B} 8, \mathrm{C} 10$ \\
4 & $440<\mathrm{CC}<460$ & 450 & $\mathrm{~A} 4, \mathrm{C} 11$ \\
5 & $481<\mathrm{CC}<505$ & 493 & $\mathrm{~A} 3, \mathrm{~B} 9, \mathrm{C} 12$ \\
\hline
\end{tabular}

CC - Consumo de cemento $\left[\mathrm{kg} / \mathrm{m}^{3}\right]$

Tabla 4. Propiedades analizadas del concreto endurecido de las mezclas

\begin{tabular}{|c|c|c|c|c|c|c|c|c|c|c|c|c|}
\hline NOMENCLATURA & $\mathrm{A} 2$ & A3 & A4 & A8 & A9 & A10 & B12 & B14 & B16 & $\mathrm{C} 20$ & $\mathrm{C} 21$ & $\mathrm{C} 25$ \\
\hline $\begin{array}{l}\text { TIPO DE } \\
\text { AGREGADO }\end{array}$ & \multicolumn{3}{|c|}{$\begin{array}{c}\text { GRAVA CALIZA Y } \\
\text { ARENA CALIIZA SIN } \\
\text { LAVAR }\end{array}$} & \multicolumn{3}{|c|}{$\begin{array}{c}\text { GRAVA CALIZA Y } \\
\text { ARENA CALIZA } \\
\text { LAVADA }\end{array}$} & \multicolumn{3}{|c|}{$\begin{array}{l}\text { GRAVA CALIZA Y } \\
\text { ARENA ANDESITA }\end{array}$} & \multicolumn{3}{|c|}{$\begin{array}{l}\text { GRAVA BASALIO Y } \\
\text { ARENA DE RIOO }\end{array}$} \\
\hline \multicolumn{13}{|c|}{ RESISTENCIA A COMPRESIÓN PARA VARIAS EDADES (DÍAS) $\left[\mathrm{kg} / \mathrm{cm}^{2}\right]$} \\
\hline & 201 & 268 & 285 & 326 & 225 & 238 & & & & 487 & 524 & 664 \\
\hline \multirow[t]{2}{*}{14} & 205 & 273 & 298 & 340 & 260 & 254 & & & & 474 & 539 & 649 \\
\hline & & & & & & & & & & 446 & 536 & 558 \\
\hline \multirow[t]{3}{*}{ PROMEDIO } & 203 & 270 & 291 & 333 & 243 & 246 & & & & 469 & 533 & 624 \\
\hline & 220 & 306 & 336 & 357 & 334 & 279 & 411 & 453 & 637 & 501 & 576 & 731 \\
\hline & 242 & 281 & 324 & 352 & 307 & 231 & & & 621 & 527 & 554 & 661 \\
\hline 28 & 224 & 293 & 320 & 344 & 325 & 269 & & & & 535 & 554 & 669 \\
\hline \multirow[t]{3}{*}{ PROMEDIO } & 229 & 293 & 327 & 351 & 322 & 260 & 411 & 453 & 629 & 521 & 561 & 687 \\
\hline & & & & & & & & & & 549 & 637 & 733 \\
\hline & & & & & & & & & & 595 & 622 & 773 \\
\hline 56 & & & & & & & & & & 528 & 554 & 776 \\
\hline \multirow[t]{3}{*}{ PROMEDIO } & & & & & & & & & & 557 & 604 & 760 \\
\hline & 270 & 328 & 371 & 389 & 354 & 279 & 470 & 563 & 758 & 628 & 598 & 748 \\
\hline & 268 & 317 & 394 & 378 & 350 & 301 & & & & 644 & 640 & 775 \\
\hline 90 & & & & & & & & & & 615 & 578 & 788 \\
\hline PROMEDIO & 269 & 322 & 382 & 383 & 352 & 290 & 470 & 563 & 758 & 629 & 605 & 770 \\
\hline 119 & & & & & & & 494 & 534 & 756 & & & \\
\hline
\end{tabular}


DOI: http://dx.doi.org/10.22201/fi.25940732e.2003.04n1.001

O. Hernández-Castañeda y A.L. Fuentes-González

Tabla 4. Propiedades del concreto endurecido (continuación)

\begin{tabular}{|c|c|c|c|c|c|c|c|c|c|c|c|c|}
\hline $\begin{array}{l}\text { NOMENCLA- } \\
\text { TURA }\end{array}$ & A2 & A3 & A4 & A8 & A9 & A10 & B12 & B14 & B16 & $\mathrm{C} 20$ & $\mathrm{C} 21$ & $\mathrm{C} 25$ \\
\hline $\begin{array}{c}\text { TIPO DE } \\
\text { AGREGADO }\end{array}$ & \multicolumn{3}{|c|}{$\begin{array}{c}\text { GRAVA CALIZA Y } \\
\text { ARENA CALIZA SIN } \\
\text { LAVAR }\end{array}$} & \multicolumn{3}{|c|}{$\begin{array}{c}\text { GRAVA CALIZA Y } \\
\text { ARENA CALIZA } \\
\text { LAVADA }\end{array}$} & \multicolumn{3}{|c|}{$\begin{array}{l}\text { GRAVA CALIZA Y } \\
\text { ARENA ANDESITA }\end{array}$} & \multicolumn{3}{|c|}{$\begin{array}{l}\text { GRAVA BASALTO Y } \\
\text { ARENA DE RIO }\end{array}$} \\
\hline \multicolumn{13}{|c|}{ MÓDULO DE ELASTICIDAD $\left[\mathrm{kg} / \mathrm{cm}^{2}\right]$} \\
\hline 28 & 2909900 & 323168 & 325216 & 312963 & 316374 & 305208 & 306256 & 320452 & 338442 & 264545 & 255432 & 292119 \\
\hline 90 & 233944 & & & & & & 333503 & 346694 & 376174 & 278690 & 276590 & 313487 \\
\hline 119 & & & & & & & 335215 & 352558 & 352778 & & & \\
\hline PROM. [28 días] & & 313095 & & & 311515 & & & 321717 & & & 270699 & \\
\hline \multicolumn{13}{|c|}{ RELACIÓN DE POISSON } \\
\hline 28 & 0.17 & 0.2 & 0.18 & 0.17 & 0.17 & 0.17 & 0.23 & 0.19 & 0.23 & 0.17 & 0.19 & \\
\hline \multicolumn{13}{|c|}{ CONTRACCIÓN POR SECADO EN VIGAS [E $\left.\mu \alpha 1^{10^{-6}}\right]$} \\
\hline 28 DÍAS & 395 & 235 & 311.25 & 338.75 & 361.25 & 312.5 & 235 & 166.25 & 245 & 287.5 & 310 & 271.25 \\
\hline 6 MESES & 565 & 395 & 457.5 & 541.25 & 577.5 & 560 & 483.75 & 376.25 & 445 & 505 & 522.5 & 501.25 \\
\hline $1 \mathrm{ANNO}$ & 627.5 & 415 & 467.5 & 576.25 & 611.25 & 560 & 528.75 & 411.25 & 471.25 & 555 & 610 & 577.5 \\
\hline \multicolumn{13}{|c|}{ COEFICIENTE DE FLUJO PLÁSTICO } \\
\hline 28 DÍAS & 1.178 & 1.5184 & 1.4261 & 1.1806 & 1.1746 & 1.297 & 0.6497 & 0.6078 & 0.7186 & 0.9357 & 0.6433 & 0.7996 \\
\hline 6 MESES & 2.2712 & 2.7279 & 2.5223 & 20.836 & 2.4167 & 2.5451 & 1.2674 & 0.9853 & 1 & 1.7089 & 1.1232 & 1.1146 \\
\hline 1 AÑO & 2.7585 & 3.2022 & 2.9966 & 2.5209 & 2.8889 & 3.1278 & 1.5802 & 1.2574 & 0.2894 & 1.8904 & 1.2865 & 1.208 \\
\hline 2 AÑOS & 3.0593 & 3.4853 & 3.2199 & 2.6849 & 3.0574 & 3.3308 & 1.8342 & 1.5098 & 1.5609 & & & \\
\hline 3 AÑOS & 3.1449 & 3.7647 & 3.4777 & 2.881 & 3.377 & 3.5489 & & & & & & \\
\hline 4 AÑOS & 3.5297 & 3.9301 & 3.6873 & & 3.6706 & 3.6504 & & & & & & \\
\hline 5 AÑOS & & & 3.8179 & & 3.7421 & 3.744 .4 & & & & & & \\
\hline ÚLTIMO & 3.4915 & 3.9743 & 3.8591 & & 3.8373 & 3.8271 & 1.8663 & 1.5123 & 1.6627 & 1.8904 & 1.2865 & 1.208 \\
\hline
\end{tabular}

diferencia en magnitud está entre la grava caliza y arena andesitica y la grava caliza y arena caliza sin lavar, con un orden próximo a $73 \%$.

La tabla 5 muestra la diferencia porcentual obtenida en la resistencia a compresión con los agregados seleccionados. El porcentaje de desarrollo promedio de todas las mezclas para 14 , $28,56,90$ y 119 días fue respectivamente igual a $89.2 \%, 100 \%, 108.1 \%$ (sólo lote C), $115.1 \%$, $118.2 \%$ (sólo lote B).

También en la curva de correspondencia entre el consumo de cemento y la resistencia a la compresión (Figura 10) se observa la influencia del consumo de cemento y del agregado en la resistencia del concreto endurecido, ya que el efecto que causa cada combinación de agregados es diferente, además de que la combinación de grava basalto y arena de río, genera la mejor resistencia a la compresión a 28 días, hecho que posiblemente se deba a que el basalto tiene un mayor módulo de elasticidad, y por lo tanto, mayor resistencia. Esto conduce a que con esta combinación de agregados, se logre un mejor aprovechamiento del consumo de cemento, en comparación con los agregados calizos. Esto se confirma al observar la eficiencia del cemento, con el nivel de resistencia a compresión (Figura 11).

El agregado tiene un papel importante en el concreto convencional ( $\left.\mathrm{f}^{\prime} \mathrm{c} \leq 400 \mathrm{~kg} / \mathrm{cm}^{2}\right)$ y para el de alta resistencia $\left(\mathrm{f}^{\prime} \mathrm{c}>400 \mathrm{~kg} / \mathrm{cm}^{2}\right)$ adquiere mayor relevancia todavía.

En las figuras 5 a 9 se observa que para un mismo o similar consumo de cemento, las combinaciones de agregados producen de mayor a menor resistencia, en el siguiente orden (Hernández, 2001):

Intervalo $1(310<\mathrm{CC}<326)$ : 
Tabla 5. Variación de la resistencia a compresión para varios agregados (en porciento)*

\begin{tabular}{ccccc}
\hline \hline & $\begin{array}{c}\text { Grava basalto y arena de } \\
\text { río }\end{array}$ & $\begin{array}{c}\text { Grava caliza y arena } \\
\text { andesita }\end{array}$ & $\begin{array}{c}\text { Grava caliza y arena } \\
\text { caliza lavada }\end{array}$ & $\begin{array}{c}\text { Grava caliza y arena } \\
\text { caliza sin lavar }\end{array}$ \\
\hline $\begin{array}{c}\text { Grava basalto y arena de } \\
\text { río }\end{array}$ & $13 \%$ & & & \\
$\begin{array}{c}\text { Grava caliza y arena } \\
\text { andesita }\end{array}$ & $55 \%$ & $41 \%$ & $13 \%$ \\
$\begin{array}{c}\text { Grava caliza y arena } \\
\text { lavada }\end{array}$ & $89 \%$ & $73 \%$ & \\
$\begin{array}{c}\text { Grava caliza y arena } \\
\text { caliza sin lavar }\end{array}$ & & $73 \%$ & \\
\hline \hline
\end{tabular}

* Porcentajes obtenidos con las mezclas analizadas y con la combinación de agregado, la cual genera menor resistencia para un mismo o similar consumo de cemento y una igual relación arena/grava. El presente cuadro, aunque fue obtenido con valores cuantitativos, pretende ser sólo cualitativo. Los valores que genere éste, sólo son valores promedio no predicciones. Por ejemplo, si se obtiene una resistencia a compresión con grava caliza y arena caliza lavada de $300 \mathrm{~kg} / \mathrm{cm}^{2}$ y se desea un valor indicativo de una mezcla con el mismo consumo de cemento, pero con agregado grueso de basalto y fino de río, el valor será $300 \times 1.89=567 \mathrm{~kg} / \mathrm{cm}^{2}$.

Mayor resistencia: grava caliza y arena caliza lavada.

Menor resistencia: grava caliza y arena caliza sin lavar

Intervalo $2(348<\mathrm{CC}<366)$ :

Mayor resistencia: grava caliza y arena andesita. Menor resistencia: grava caliza y arena caliza lavada.

Intervalo $3(380<\mathrm{CC}<401)$ :

Mayor resistencia: grava basalto y arena de río. grava caliza y arena andesita.

Menor resistencia: grava caliza y arena caliza sin lavar.

Intervalo $4(440<\mathrm{CC}<460)$ :

Mayor resistencia: grava basalto y arena de río.

Menor resistencia: grava caliza y arena caliza lavada.

Intervalo $5(481<\mathrm{CC}<505)$ :

Mayor resistencia: grava basalto y arena de río. grava caliza y arena andesita.

Menor resistencia: grava caliza y arena caliza sin lavar.

En resumen, los agregados presentan un mejor desarrollo de resistencia a compresión en el siguiente orden (de mayor a menor resistencia):
1. Grava basalto y arena de río.

2. Grava caliza y arena andesita.

3. Grava caliza y arena caliza lavada

4. Grava caliza y arena caliza sin lavar

Se aprecia la diferencia en el desarrollo de la resistencia para dos concretos elaborados con grava caliza, pero diferentes arenas; el primero con arena caliza y el segundo con arena andesitica, en donde presentó mayor resistencia el concreto con arena andesitica.

Hasta ahora se ha discutido solamente el efecto del tipo de agregado en la resistencia a compresión del concreto; a continuación, se plantea el efecto que tiene en la deformabilidad del concreto a corto y largo plazo.

\section{Módulo de Young}

Los valores obtenidos se muestran en la tabla 4. En la figura 12 se presenta la relación resistencia a compresión - módulo de Young, donde se aprecia el efecto del tipo de agregado en las propiedades del concreto, y se compara con la relación indicada en el RCDF, para concreto clase 1.

\section{Flujo plástico}

Como la mayoría de los materiales estructurales, el concreto se deforma instantáneamente al 
aplicarle una carga por primera vez, pero ante una carga ininterrumpida o constante, el concreto presenta flujo plástico.

E1 flujo plástico, llamado también deformación diferida, deformación plástica o fluencia, es el incremento de deformación del concreto, debido a una carga sostenida en el tiempo.

Es decir, se presenta un incremento en la deformación bajo un esfuerzo constante e ininterrumpido, que incluye las deformaciones vinculadas con el esfuerzo y excluye las causadas por contracción por secado, deformaciones térmicas y de inflamiento; por ello, el flujo plástico se considera a partir de la deformación inicial generada por el módulo de Young a la edad de carga.

El concreto presenta una deformación inmediata o inicial [ $\varepsilon$ i] y otra diferida [flujo plástico, $\varepsilon_{d}$ ] La deformación inicial debida a la carga que soporta el concreto, es la deformación instantánea y depende fundamentalmente de la proporción del esfuerzo aplicado, tiene pocos efectos dependientes del tiempo, mientras que la deformación adicional, causada por la misma carga, es la deformación por flujo plástico y depende de la proporción del esfuerzo aplicado y del tiempo de aplicación del esfuerzo.

Por lo tanto, para evaluar la deformación del concreto bajo carga sostenida se debe considerar el tiempo que dura la aplicación de la carga y el descuento en las deformaciones no estructurales, como la contracción por secado, que es una deformación autógena que todo concreto presenta, ante la progresiva pérdida de humedad (Neville, 1992; Neville y Brooks, 1998).

La deformación total de un concreto se debe entonces a la deformación instantánea, al flujo plástico y a la contracción, como lo indica la ecuación 1.

$$
\begin{gathered}
\text { Deformación total }\left(\varepsilon_{\mathrm{T}}\right)=\text { Deformación } \\
\text { instantánea }\left(\varepsilon_{\mathrm{E}}\right)+\text { Flujo plástico }\left(\varepsilon_{\mathrm{FP}}\right)+ \\
\text { Contracción }\left(\varepsilon_{\mathrm{c}}\right)
\end{gathered}
$$

El flujo plástico no puede observarse en forma directa y sólo se puede determinar al restar la deformación inicial y la deformación por contracción por secado, a la deformación total.

El flujo plástico del concreto bajo carga sostenida es un proceso lento que puede prolongarse indefinidamente, por lo que se considera que está presente en todo la vida del concreto y en cualquier estructura.

Para que el flujo plástico no represente sólo una pequeña deformación de poco interés práctico, se debe hacer notar que una deformación de flujo plástico típica después de un año de carga, es varias veces mayor a la deformación inicial. A continuación, en la tabla 6 se presenta un ejemplo con valores numéricos de deformaciones que son resultado de los tres factores mencionados anteriormente para los valores promedio obtenidos de los especímenes en esta investigación; así como para un espécimen de concreto normal sujeto a $63.3 \mathrm{~kg} / \mathrm{cm}^{2}$ en compresión.

Con estos valores relativos, se hace evidente que las relaciones esfuerzo-deformación para cargas de corta duración, pierden importancia ante las cargas de larga duración por tener un efecto más dominante en el comportamiento de una estructura.

Tabla 6. Importancia del flujo plástico en la deformación total del concreto

Tipo de deformación

Deformación elástica o inmediata

Deformación por contracción al año $\varepsilon_{\mathrm{SH}}=$

Deformación por flujo plástico al año $\varepsilon_{\mathrm{SH}}=$

Deformación total al año $\varepsilon_{\mathrm{T}=}$

Valores promedio obtenidos de nuestra investigación (varios especímenes sujetos a $0.4 \mathrm{fc}$ )

$668 \times 10^{-6} \mathrm{~m}-\mathrm{m}$

$562 \times 10^{-6} \mathrm{~m}-\mathrm{m}$

$1296 \times 10^{-6} \mathrm{~m}-\mathrm{m}$

$2526 \times 10^{-6} \mathrm{~m}-\mathrm{m}$

Especímen de concreto normal sujeto a $900 \mathrm{psi}\left(63.3 \mathrm{~kg} / \mathrm{cm}^{2}\right)$ en compresión

$250 \times 10^{-6}$ in-in

$500 \times 10^{-6}$ in-in

$750 \times 10^{-6}$ in-in

$1500 \times 10^{-6}$ in-in 
En resumen, la importancia del flujo plástico en el concreto estructural, radica principalmente en que la deformación por flujo plástico tiene un orden mayor de magnitud que la deformación inicial. La determinación del flujo plástico es relevante para el cálculo de esfuerzos cuando ocurre la relajación, por ejemplo, para el concreto presforzado, y para el cálculo de deformaciones y desplazamientos en miembros estructurales.

El flujo plástico se determinó experimentalmente para los tres lotes de concreto, la edad mínima de determinación experimental fue de un año. En la predicción del flujo plástico se utilizan los métodos de Neville y de Fuentes, porque son métodos que se basan en datos experimentales.

El método Neville propone la siguiente ecuación:

$$
C_{T}=\frac{t^{0.6}}{b+t^{0.6}} C_{U}
$$

Por su parte el método Fuentes propone:

$$
C_{T}=\frac{t^{a}}{b+t^{a}} C_{U}
$$

Donde:

$\mathrm{C}_{\mathrm{T}}$ es el coeficiente de flujo plástico.

$\mathrm{C}_{u}$ es el coeficiente último de flujo plástico,

t es la edad del concreto a partir de que es cargado. b (en días) y a son constantes propias de cada concreto; son función del tiempo de carga sostenida.

Para determinar las variables $a, b$ y $C_{u}$ se realiza un análisis estadístico y matemático de las lecturas de deformación registradas en los especímenes colocados en el marco de flujo plástico y de los testigos sin carga, con el equipo whittemore.

Normatividad vigente del flujo plástico

Las Normas Técnicas Complementarias de Estructuras de Concreto del Reglamento de Construcciones del Distrito Federal (RCDF) establecen que se debe considerar un coeficiente de flujo plástico o deformación diferida final de 2.4 para concretos clase 1 (peso específico mayor a $2.2 \mathrm{t} / \mathrm{m}^{3}$ ) y de 5 para concreto de clase 2 (peso específico comprendido entre 1.9 y $2.2 \mathrm{t} / \mathrm{m}^{3}$ ).

\section{Análisis de datos experimentales}

Para las doce mezclas de concreto analizadas, se determinó experimentalmente el flujo plástico por un período mínimo de un año y se aplicó el método de predicción señalado. El desarrollo del flujo plástico, real y estimado para las combinaciones de agregado propuestas, se presenta en las gráficas 13 a 16 .

\begin{tabular}{|c|c|c|c|c|c|c|}
\hline \multirow{2}{*}{$\begin{array}{c}\text { TIPO DE } \\
\text { AGREGADO }\end{array}$} & \multicolumn{3}{|c|}{$T=1$ año } & \multicolumn{3}{|c|}{$T=2$ años } \\
\hline & Neville & Fuentes & $\mathrm{C}_{\mathrm{T}}$ & Neville & Fuentes & $\mathrm{C}_{\mathrm{T}}$ \\
\hline $\begin{array}{l}\text { Grava basalto y } \\
\text { arena de río }\end{array}$ & $C_{T}=\frac{t^{0.6}}{10.2+t^{0.6}} 2.0$ & $C_{T}=\frac{t^{0.66}}{13.4+t^{0.66}} 1.9$ & 1.5 & $C_{T}=\frac{t^{0.6}}{8.7+t^{0.6}} 1.6$ & $C_{T}=\frac{t^{0.6}}{13.7+t^{0.6}} 1.8$ & 1.6 \\
\hline $\begin{array}{l}\text { Grava caliza y } \\
\text { arena andesita }\end{array}$ & $C_{T}=\frac{t^{0.6}}{17.6+t^{0.6}} 2.1$ & $C_{T}=\frac{t^{0.72}}{20.2+t^{0.72}} 1.8$ & 1.4 & $C_{T}=\frac{t^{0.6}}{21.1+t^{0.6}} 2.3$ & $C_{T}=\frac{t^{0.65}}{23.5+t^{0.65}} 2.1$ & 1.6 \\
\hline $\begin{array}{l}\text { Grava caliza y } \\
\text { arena caliza lavada }\end{array}$ & $C_{T}=\frac{t^{0.6}}{22.5+t^{0.6}} 4.8$ & $C_{T}=\frac{t^{0.72}}{23.0+t^{0.72}} 3.6$ & 2.8 & $C_{T}=\frac{t^{0.6}}{19.73+t^{0.6}} 4.4$ & $C_{T}=\frac{t^{0.75}}{24.8+t^{0.75}} 3.6$ & 3.0 \\
\hline $\begin{array}{l}\text { Grava caliza y } \\
\text { arena caliza sin } \\
\text { lavar }\end{array}$ & $C_{T}=\frac{t^{0.6}}{17.0+t^{0.6}} 4.4$ & $C_{T}=\frac{t^{0.68}}{17.4+t^{0.6}} 3.8$ & 3.0 & $C_{T}=\frac{t^{0.6}}{16.2+t^{0.6}} 4.3$ & $C_{T}=\frac{t^{0.7}}{18.6+t^{0.7}} 3.8$ & 3.3 \\
\hline
\end{tabular}

Tabla 7. Valores experimentales y ecuaciones promedio de flujo plástico 
En la tabla 7 se muestran los valores experimentales y los parámetros de predicción para los métodos de flujo plástico.

Para los concretos elaborados con distintos tipos de agregados la forma de la curva de flujo plástico resultó muy similar.

Al comparar las mezclas de concreto elaboradas con el mismo tipo de agregado, pero diferente consumo de cemento y relación agua/cemento, se aprecia que no necesariamente hay una relación entre consumo de cemento y flujo plástico o relación agua/cemento - flujo plástico para las mezclas analizadas. Tanto el consumo de cemento, como la relación agua/cemento, tienen un doble efecto en el desarrollo del flujo plástico. Por un lado, a mayor consumo de cemento o menor relación agua/cemento, se tiene un concreto con mayor resistencia y menor deformabilidad, lo que tendría por consecuencia una disminución del flujo plástico; por otro lado, al tener mayor consumo de cemento o menor relación agua/cemento, se logra un mayor volumen de la pasta de cemento, que es la fuente del flujo plástico, por lo que se incrementaría éste último. En este sentido, se encontró una relación sólo para los concretos con grava basáltica y arena de río, donde a mayor consumo de cemento y menor relación agua/cemento se obtienen un menor coeficiente de flujo plástico.

El efecto del consumo de cemento en el coeficiente de flujo plástico se muestra en la figura 17, donde se señalan las diferencias de emplear un agregado u otro, de modo que un concreto con $400 \mathrm{~kg} / \mathrm{m}^{3}$ denotará un menor coeficiente de flujo plástico si lo constituyen agregados gruesos de basalto y finos de río, o grava caliza y arena andesita, por lo que presentará un mayor coeficiente si contiene arena y grava caliza. Por su parte, las deformaciones totales del concreto son afectadas por el tipo de agregado (tabla 8).

Los valores anteriores se muestran en la figura 18 , en la cual se confronta la deformación total contra el consumo de cemento y permite apreciar como los agregados gruesos calizos restringen en mayor magnitud la deformación total que el agregado basalto. Así, los concretos con ambos agregados calizos tienen coeficientes de flujo plástico altos, pero deformaciones totales moderadas, mientras que un concreto con agregado fino de río y grueso de basalto desarrolla deformaciones totales de mayor magnitud y coeficientes de flujo plástico bajos.

En primera instancia, los resultados anteriores parecen contradictorios; sin embargo, tienen una explicación perfectamente lógica: los agregados calizos en el concreto provocan deformaciones elásticas de baja magnitud y los concretos con grava de basalto y arena de río provocan deformaciones iniciales del orden del doble que los calizos.

Los concretos con grava caliza y arena andesitica desarrollan deformaciones iniciales intermedias de las combinaciones de agregados anteriores, la deformación elástica adquiere relevancia porque el coeficiente de flujo plástico está en función de ella.

Al utilizar los mismos intervalos de consumo de cemento señalados al principio, se comparó el efecto del agregado en el flujo plástico, para un mismo o similar consumo de cemento.

En las gráficas 19 a 23 se presenta dicha comparación, donde se observa que la combinación de agregados restringe el desarrollo del flujo plástico del concreto de la siguiente manera (Hernández, 2001):

Tabla 8. Deformación total de acuerdo al tipo de agregado

Agregado en el concreto

Grava caliza y arena caliza sin lavar

Grava caliza y arena caliza lavada

Grava caliza y arena andesita

Grava basalto y arena de río
Deformación total promedio (1 año)

$2252.9 \times 10^{-6}$

$2277.3 \times 10^{-6}$

$2245.1 \times 10^{-6}$

$3322.4 \times 10^{-6}$ 
Intervalo 1:

Menor fp: grava caliza y arena caliza sin lavar Mayor fp: grava caliza y arena caliza lavada

\section{Intervalo 2:}

Menor fp: grava caliza y arena andesita Mayor fp: grava caliza y arena caliza lavada Intervalo 3:

Menor fp: grava caliza y arena andesita grava basalto y arena de río

Mayor fp: grava caliza y arena caliza sin lavar

\section{Intervalo 4:}

Menor fp: grava basalto y arena de río Mayor fp: grava caliza y arena caliza lavada

Intervalo 5:

Menor fp: grava basalto y arena de río grava caliza y arena andesita

Mayor fp: grava caliza y arena caliza sin lavar

En donde fp es Flujo plástico.

En términos generales los agregados restringen al flujo plástico del concreto en el siguiente orden (de menor a mayor flujo plástico generado):

1. Grava basalto y arena de río.

2. Grava caliza y arena andesita.

3. Grava caliza y arena caliza lavada

4. Grava caliza y arena caliza sin lavar

La restricción que ejercen la combinación basalto-arena de río y caliza-andesita, tiende a valores similares del coeficiente del flujo plástico, y muestra una ligera ventaja en la combinación basalto-arena de río. El efecto del lavado en arenas calizas es mínimo en el flujo plástico.

Determinar el efecto del agregado en el flujo plástico implica mayor dificultad, debido a la variedad de factores que actúan activamente en el flujo plástico: En general, el agregado grueso de basalto y la arena de río, así como la grava caliza y la arena andesitica restringen en mayor magnitud flujo plástico, en comparación con aquellos con agregados calizos.

\section{Conclusiones}

El empleo del agregado en el concreto es de suma importancia, debido a que influye activamente para un mejor comportamiento: genera mayores resistencias a la compresión que para un mortero. El agregado grueso restringe al flujo plástico y a la contracción por secado, así que en términos generales, a mayor volumen de agregado, menor flujo plástico y contracción por secado.

Según sea el tipo y calidad del agregado, el concreto tendrá mejores propiedades de resistencia, módulo de Young, flujo plástico y contracción por secado. Por ello, es importante considerar el tipo de agregado en la proporción de las mezclas.

El incremento de resistencia es proporcional al incremento de consumo de cemento, pero dicho incremento depende también del tipo de agregado empleado.

Los concretos con ambos agregados calizos muestran un desarrollo moderado de resistencia, entre 229 y $352 \mathrm{~kg} / \mathrm{cm}^{2}$ a 28 días, para consumos de cemento de 317 a $491 \mathrm{~kg} / \mathrm{m}^{3}$, y un coeficiente alto de flujo plástico, que varia entre 2.52 y 3.12 y una contracción por secado de 447 a 563 X10.6 al año, por lo que su aplicación debe enfocarse a estructuras de baja tecnología, donde no se requieren altas resistencias a compresión o tensión (edificios de poca altura y/o edificaciones en zonas de bajo riesgo sísmico).

Los concretos elaborados con grava de basalto y arena de río presentan un excelente desarrollo de resistencia, ya que con consumos de cemento entre 400 y $500 \mathrm{~kg} / \mathrm{m}^{3}$ se obtienen resistencias entre 521 y $687 \mathrm{~kg} / \mathrm{cm}^{2}$, coeficientes de flujo plástico al año entre 1.28 y 1.89 y una contracción por secado importante (entre 707 y 817 X10 $0^{-6}$ ).

Con tales características, el concreto con grava basáltica y arena de río puede aplicarse a estructuras donde se requiera alta resistencia, y se consideren las medidas adecuadas para restringir en lo posible la contracción por secado. Su aplicación en estructuras hidráulicas es perfectamente viable, por su resistencia y porque en estructuras hidráulicas como canales de descarga no hay cargas sostenidas (no se presentará flujo plástico), y porque con un adecuado curado y con el flujo por el canal de agua 
(aunque este sea ocasional) se restringe la contracción por secado. Una buena práctica es humedecer el concreto en temporadas con altas temperaturas ambientales.

El agregado grueso de basalto y la arena de río, han mostrado en este estudio ser agregados alternos viables para la producción de concreto; es necesario investigar a fondo su viabilidad para estructuras de gran importancia. Con sus características benéficas encontradas en el ámbito de resistencia del concreto y sus características de deformabilidad, resulta ser una combinación alterna de agregados perfectamente utilizable para estructuras de baja y media tecnología. Para su aplicación en estructuras de mayor importancia, donde se requiere un grado tecnológico considerable, es necesario encontrar una solución para emplear grava de origen basáltico y obtener un concreto con baja deformabilidad.

Los concretos con grava caliza y arena andesita poseen un buen desarrollo de resistencia a compresión (entre 411 y 629 kg/ $\mathrm{cm}^{2}$ ) para consumos de cemento que oscilan entre 350 y 493 $\mathrm{kg} / \mathrm{m}^{3}$ y un moderado coeficiente flujo plástico (entre 1.25 y 1.58 para un año) y una contracción por secado entre 407 y $537 \times 10^{-6}$, por lo que su aplicación es universal, ya que ofrece resistencias altas con consumo de cementos relativamente moderados y baja deformabilidad a largo y corto plazo; de hecho es la combinación de agregados que ofrece menor deformabilidad total y un bajo coeficiente de flujo plástico, algo similar sucede en la contracción por secado, donde presenta menor promedio; en suma, es un concreto con muy buenas características de resistencia y deformabilidad, por lo que es una excelente opción para elaborar concreto en el Valle de México.

Existe un mejor aprovechamiento del incremento de consumo de cemento, tanto para la grava basáltica y la arena de río, como para la grava caliza y la arena andesitica para ambos agregados calizos.

Tanto el agregado grueso basáltico y la arena de río como el agregado grueso calizo y fino de andesita, generan mayores resistencias que la grava y arena caliza para un mismo consumo de cemento.

El flujo plástico es una propiedad importante del concreto de largo plazo que debe investigarse con mayor profundidad. Es un nexo entre las propiedades de resistencia y deformabilidad con poco desarrollo; así como una escasa normatividad en México. En la medida que las estructuras y miembros de concreto incluyan este factor, se incrementará la confiabilidad de las estructuras y se evitarán efectos dañinos.

El RCDF propone sólo dos valores generales, según sea la clase de concreto, sin incluir una estimación abierta y por lo tanto más adecuada paraconcretos con características particulares, como concretos de alto desempeño, masivos, ligeros o para concretos cargados a distintas edades, compactados y curados con distintos métodos, así como para diversas condiciones de servicio.

Es conveniente realizar una revisión cuidadosa y a detalle, con el fin de proponer un modelo confiable de predicción del flujo plástico en el concreto, que considere todas las variables involucradas en este singular fenómeno, presente en prácticamente cualquier estructura de concreto. En la medida que nuestra normatividad técnica, conocimiento y práctica constructiva sean compatibles, se podrá aspirar a mejorar la ingeniería civil mexicana.

Es necesario y de vital importancia para la ingeniería mexicana, buscar agregados alternos, así como incentivar su investigación y posterior empleo en el concreto.

El conocimiento del efecto del agregado en el concreto, proporciona al ingeniero civil, ya sea diseñador estructural o ingeniero constructor, una valiosa herramienta para determinar las propiedades de resistencia y deformabilidad del concreto. Con la publicación de este artículo se pretende hacer un llamado a los ingenieros civiles para que conozcan y posean un conocimiento más amplio de lo que es fabricar y construir profesionalmente con concreto. 


\section{Agradecimientos}

Este trabajo se realizó en el Laboratorio de Estructuras y Materiales del Instituto de Ingeniería, UNAM. Agradecemos al Maestro C.J. Mendoza, así como al personal directivo y operativo por su colaboración para realizar esta investigación.

Al M.I. Francisco Téllez Granados por la revisión técnica del escrito y al Consejo Nacional de Ciencia y Tecnología (CONACYT) por su apoyo moral y económico al primer autor.

\section{Referencias}

ACI 209R - 92 (Reaproved 1997). Prediction of Creep, Shrinkage, a And EmperatureEffects Inc Oncrete Structures. ACI. Manual of Concrete Practice 2000, Part 1, Materials and General Properties of Concrete.

Hernández-Castañeda O. (2001). Actualización de ecuaciones de flujo plástico para diversos concretos. Tesis de Licenciatura en Ingeniería Civil, Universidad Nacional Autónoma de México, Ciudad Universitaria, México.

Mendoza C.J., Carmona C.M, Fuentes G.A. (1995). Concretos fabricados con arenas y gravas calizas disponibles en el DF. Informe preparado para la Secretaría General de Obras del Departamento del Distrito Federal. Instituto de Ingeniería, UNAM, México

Mendoza C.J., Fuentes G.A. y Aire U.C. (1993). Concretos estructurales con agregados basálticos. Informe preparado para Grupo Corporativo Interestatal SA de CV. Instituto de Ingeniería, UNAM, México.

Neville A.M. y Brooks I.J. (1998). Tecnología del concreto. Traducción de Concrete Technology. Ed. Trillas, México.

Neville A.M. (1992). Tecnología del concreto, traducción de Propperties of Concrete. Instituto Mexicano del Cemento y del Concreto AC, México.

Rico-Rodríguez A. y Del Castillo H.(2000). La Ingeniería de suelos en las vías terrestres. Ed. Limusa, México.

\section{Bibliografía sugerida}

ACI 211.1-91 Standard Practice for Selecting Proportions for Normal, Heavyweight, a And Mass Concrete. ACI. Manual of Concrete Practice 2000, Part 1, Materials and General Properties of Concrete.

Aitcin P.C. (2001). The Future of the Cement and Concrete Industry at the Dawn of the Century. Cátedra Cemex. Ponencia sustentada en la Facultad de Ingeniería, UNAM. Abril.

Aitcin P.C. (1998). High-Performance Concrete, Modern Concrete Technology 5. E \& Fn Spon, Usa \& Canada.

Alexander M.G. (1996). Aggregate S and the Deformation Properties of Concrete. CIi ACI Materials Journal, V.93, No.6, November-December.

ASTM Volume 04.02 Concrete and Concrete Aggregates, Annual Book of Astm Standars (1998). American Society for Testing and Materials.

ASTM Volume 04.03 Cement and Gypsum 1998, Annual Book Of Astm Standars (1998). American Society for Testing and Materials.

Bazant Z.P. y. Baweja. S. (1994). Concrete Creep and Shinkage Prediction Models for Design Codes. En: Concrete Technology: New Trends, Industrial Applications. (Proceeding of The International RILEM Workshop (26). E \& Fn Spon, Barcelona, España.

Cetin A. y Carrasquillo R.L. (1998). High-Performance Concrete:Influence of Coarse Aggregates on Mechanical Properties. ACI Materials Journal, V.95, No.3, May-June.

Collins T.M. (1989). Proporcioning High-Strength Concrete to Control Creep and Shrinkage. ACI Materials Journal, V.86, No.6, November-December.

Comisión Federal de Electricidad-Instituto de Ingeniería, UNAM. (1994). Manual de tecnología del concreto, Sección 3. Ed. Limusa, México.

Departamento de Ingeniería Experimental-Secretaría de Recursos Hidráulicos (1970). Manual de Concreto. Parte 2.México.

Gaceta Oficial del Distrito Federal (1996). Normas técnicas complementarias para diseño y construcción de estructuras de concreto del reglamento de construcciones para el Distrito Federal, Marzo. 
Gardner N.J. y Zhao J.W. (1993). Creep and Shrinkage Revisited. ACI Materials Journal, V.90, No.3, May-June.

Kosmatka S.H. (1999). Concrete Construction for the Next Millenium. Primer Congreso Interamericano del Cemento y del Concreto, Ciudad de México, Septiembre.

Kosmatka S.H. y Panarese W.C. (1992). Diseño y control de mezclas de concreto. Portland Cement Association. Edición y traducción al español del Instituto Mexicano del Cemento y del Concreto AC, México.

Mendoza Carlos J., Aire U.C., Contreras P. y Fuentes G.A.(1998). Concretos de alto desempeño con materiales comunes en el Distrito Federal. Informe preparado para la Secretaria General de Obras del Departamento del Distrito Federal. Instituto de Ingeniería, UNAM, Diciembre, México.
Nawy EG. (1988). Concreto reforzado, un enfoque básico. Prentice Hall, México.

Neville A. (2000). Tecnología del concreto, un elemento esencial del diseño estructural. Construcción y Tecnología, Instituto Mexicano del Cemento y del Concreto AC.

Neville A. (1997). Aggregate Bond and Modulus of Elasticity of Concrete. ACI Materials Journal, V.94, No.1, January-February.

Neville A.M. (1970). Creep Of Concrete: Plain, Reinforced and Prestresed. North Holland Publishing Company. Amsterdam.

Popovics S. (1990). Analysis of the Concrete Strength Versus Water-Cement Ratio Relationship. ACI Materials Journal, V.87, No.5, September-October.

Ross A.D.(1958). Creep of Concrete Under Variable Stress. Journal of the American Concrete Inst itute, V.29, No.9, Proceedings V. 54, March. 


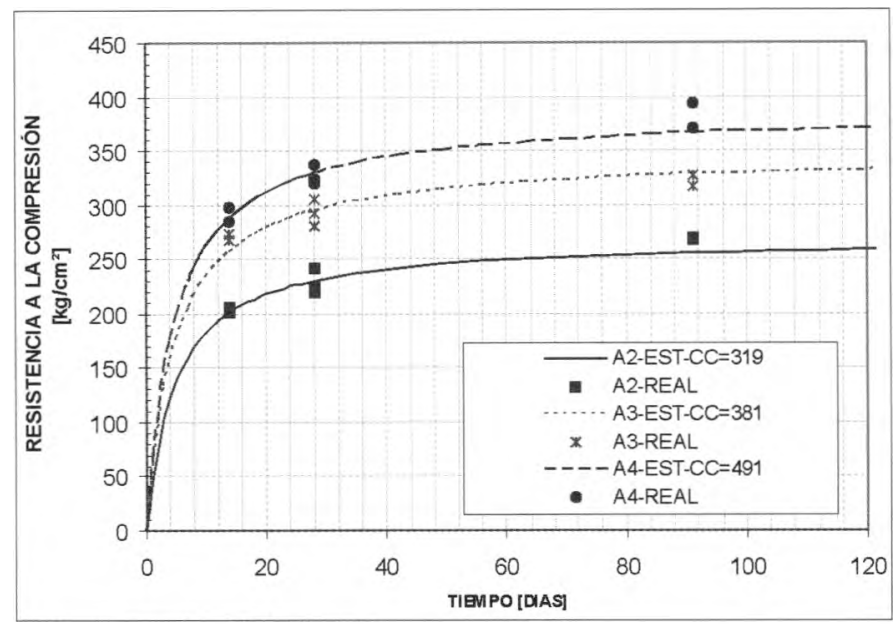

Figura 1. Desarrollo de la resistencia a la compresión de concretos con grava caliza y arena caliza sin lavar

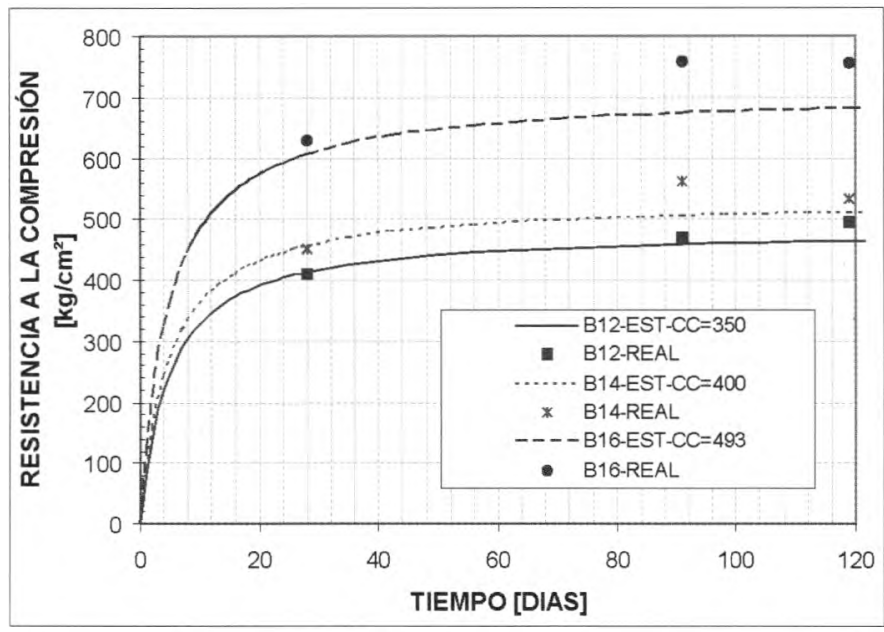

Figura 3. Desarrollo de la resistencia a la compresión de concretos con grava caliza y arena andesita

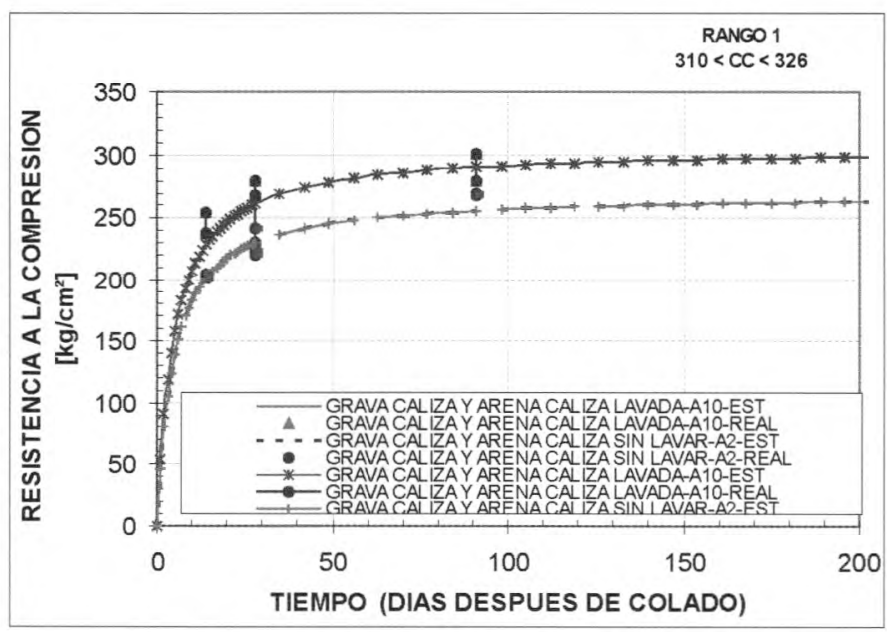

Figura 5. Desarrollo de la resistencia a la compresión de concretos fabricados con diferentes agregados

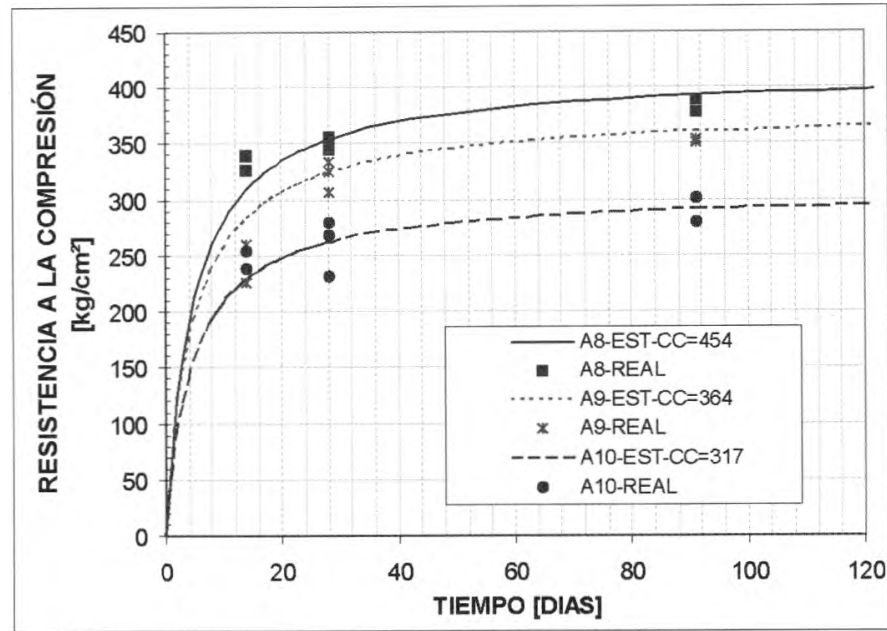

Figura 2. Desarrollo de la resistencia a la compresión de concretos con grava caliza y arena caliza lavada

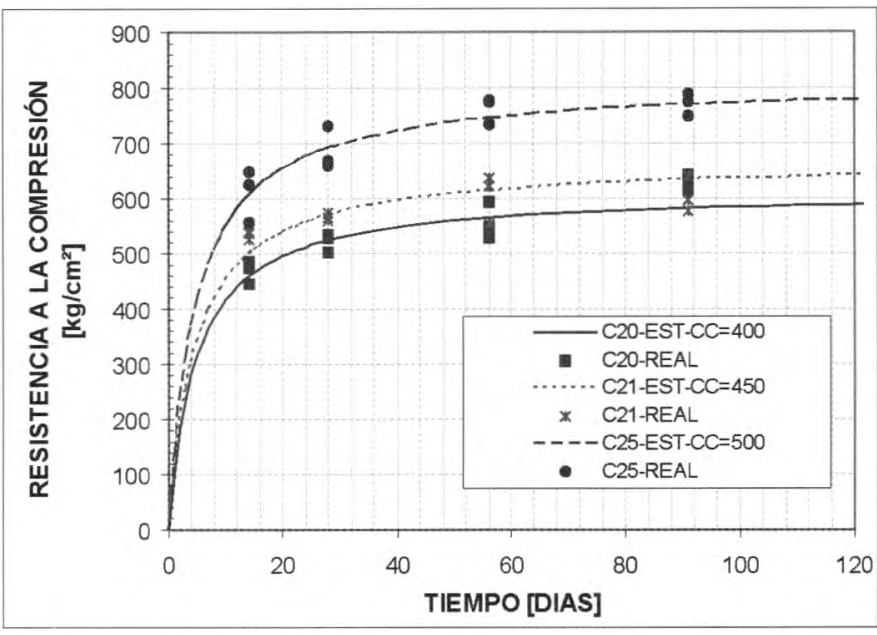

Figura 4. Desarrollo de la resistencia a la compresión de concretos con grava basalto y arena de río

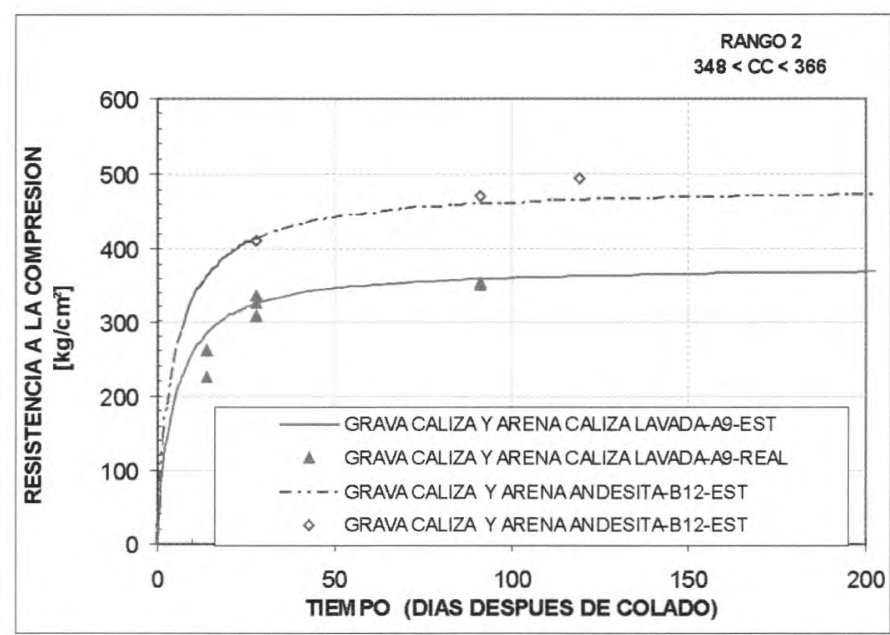

Figura 6. Desarrollo de la resistencia a la compresión de concretos fabricados con diferentes agregados 
DOI: http://dx.doi.org/10.22201/fi.25940732e.2003.04n1.001

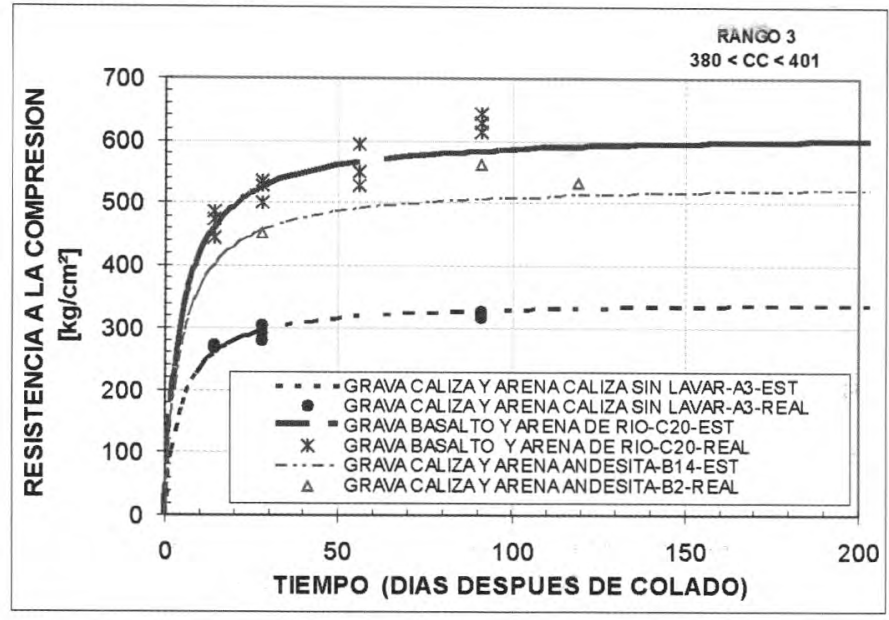

Figura 7. Desarrollo de la resistencia a la compresión de concretos fabricados con diferentes agregados

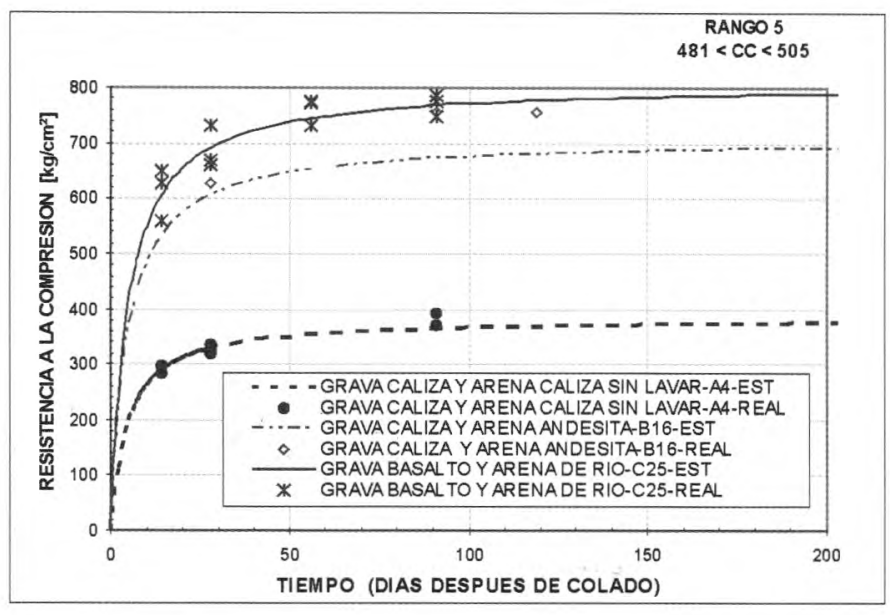

Figura 9. Desarrollo de la resistencia a la compresión de concretos fabricados con diferentes agregados

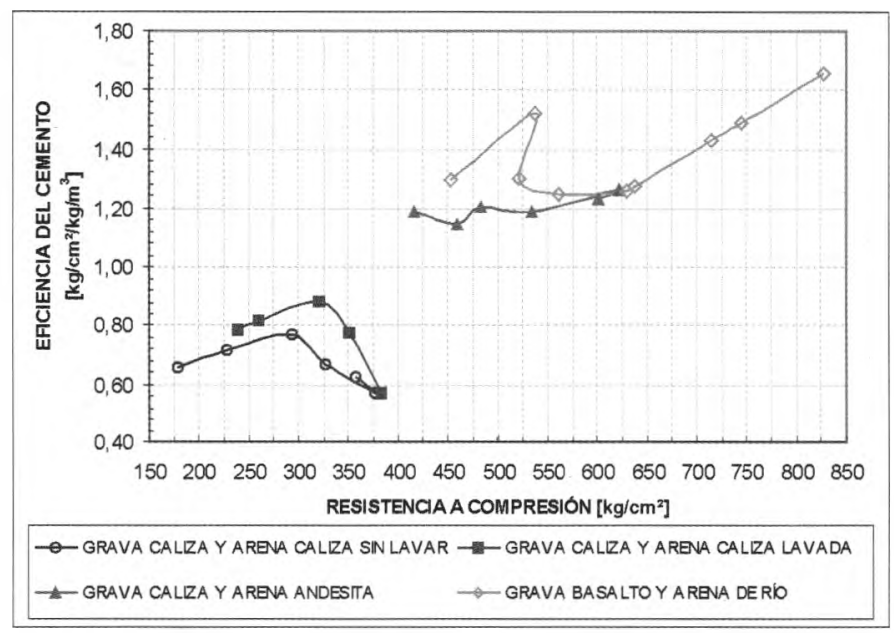

Figura 11. Eficiencia del cemento con el nivel de la resistencia a la compresión para varios agregados

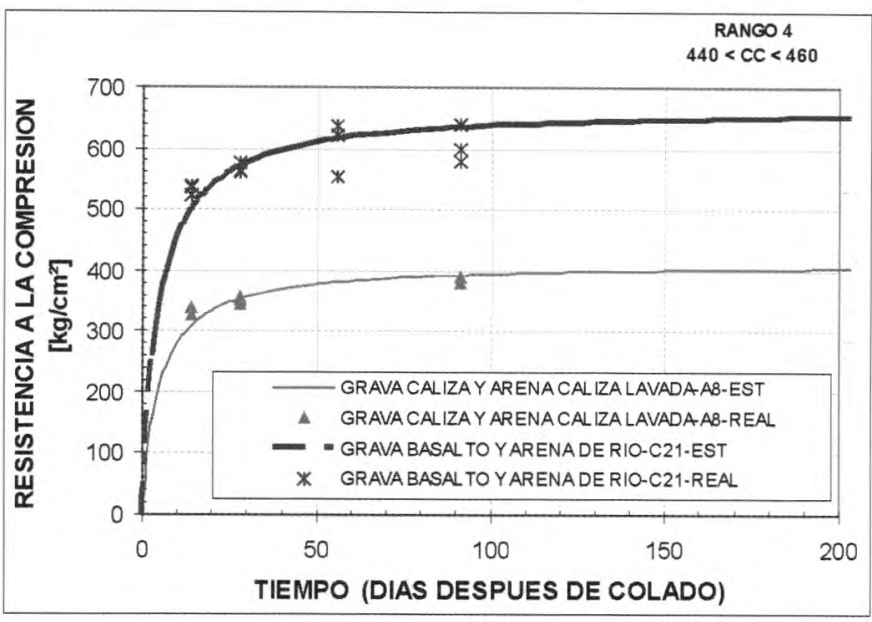

Figura 8. Desarrollo de la resistencia a la compresión de concretos fabricados con diferentes agregados

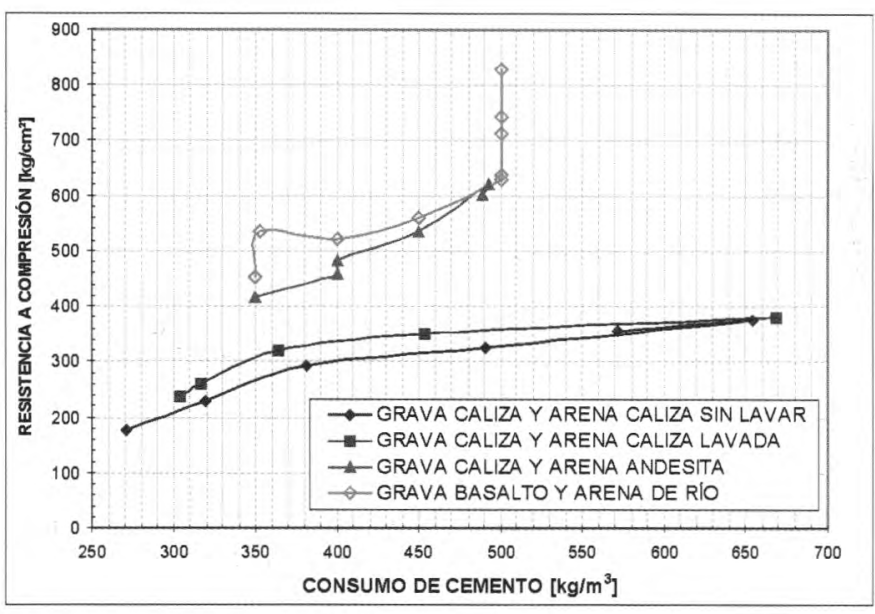

Figura 10. Curva consumo de cemento - resistencia a la compresión para varios agregados

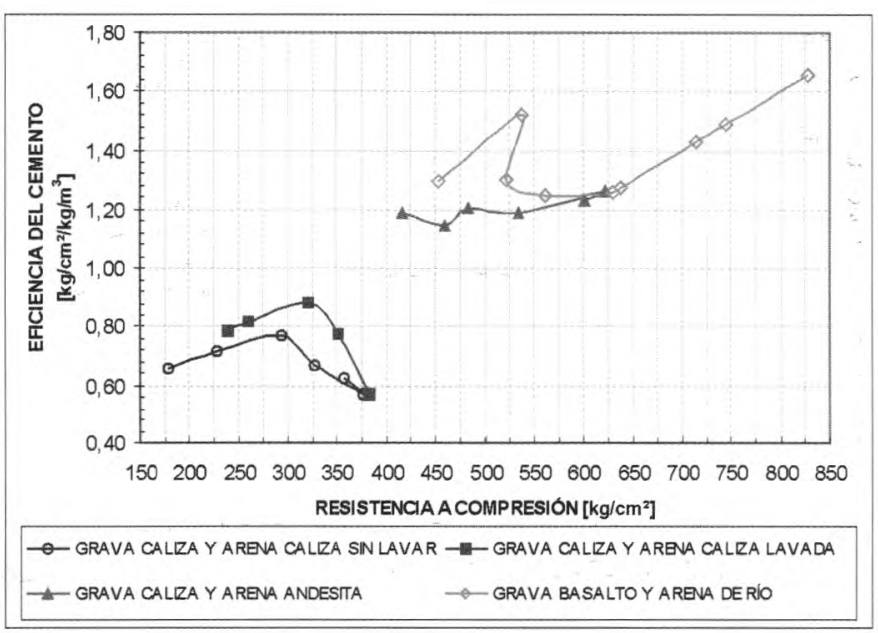

Figura 12. Relación resistencia a compresión - módulo de elasticidad para varios agregados (28 dias) 


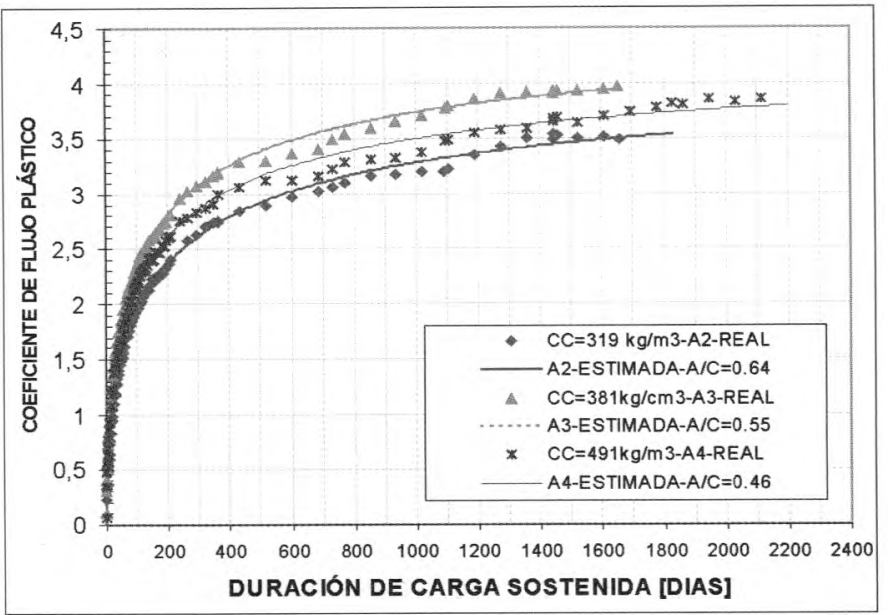

Figura 13. Desarrollo del flujo plástico de concretos con grava caliza y arena caliza sin lavar

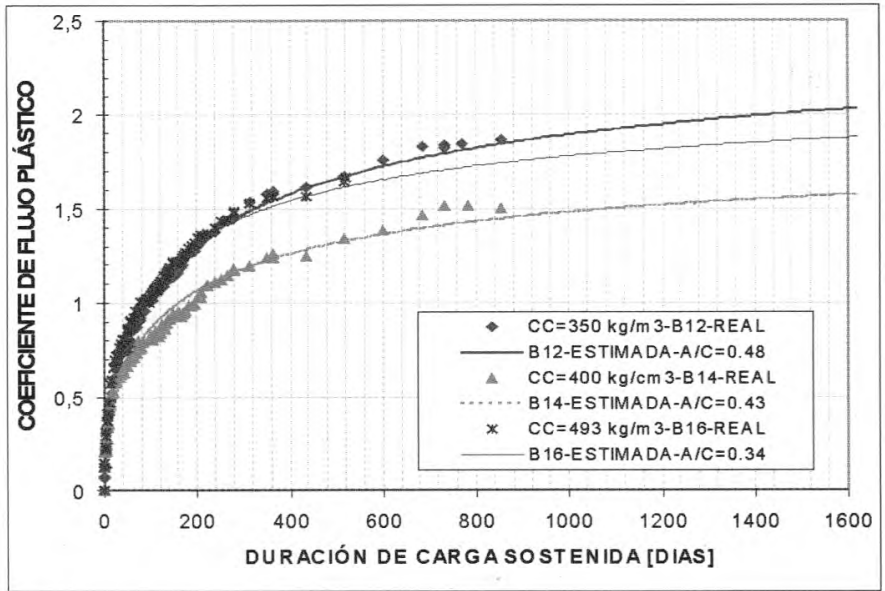

Figura 15. Desarrollo del flujo plástico de concretos con grava caliza y arena andesita

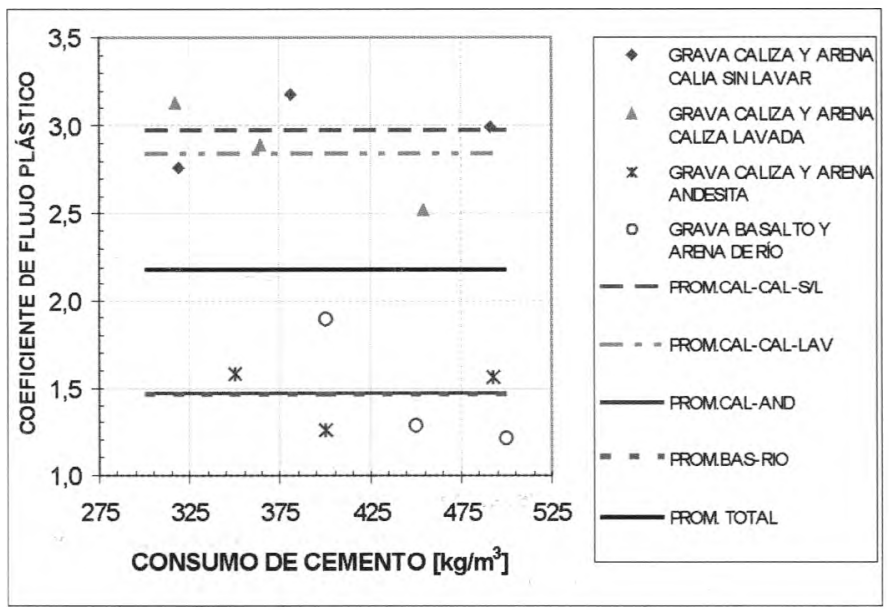

Figura 17. Efecto del consumo de cemento en el coeficiente de flujo plástico (1 año)

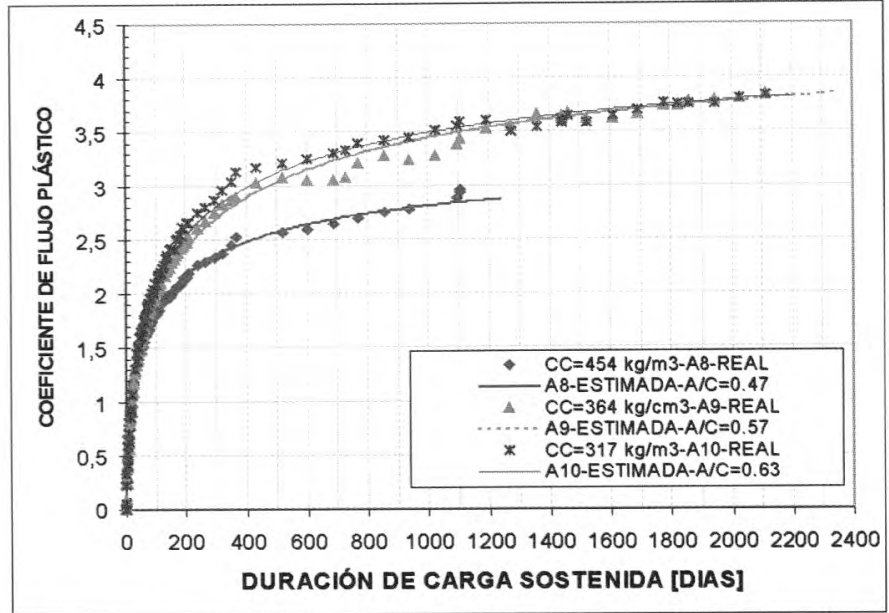

Figura 14. Desarrollo del flujo plástico de concretos con grava caliza y arena caliza lavada

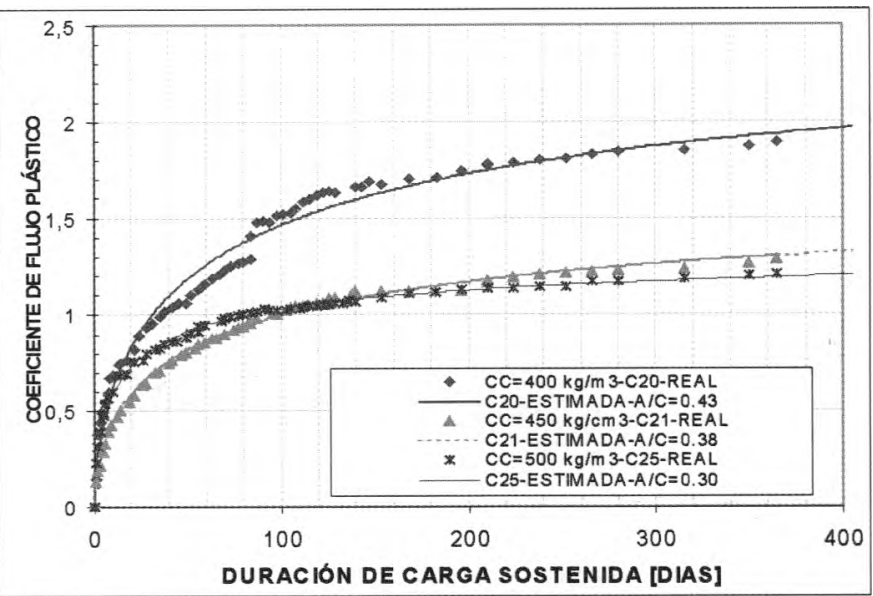

Figura 16. Desarrollo del flujo plástico de concretos con grava, basalto y arena de río

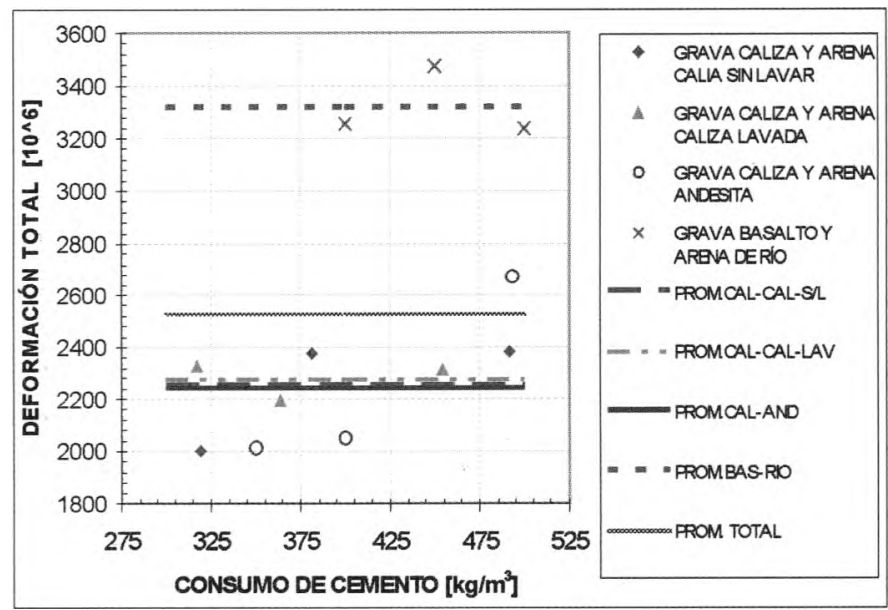

Figura 18. Efecto del consumo de cemento en la deformación total del concreto (1 año) 


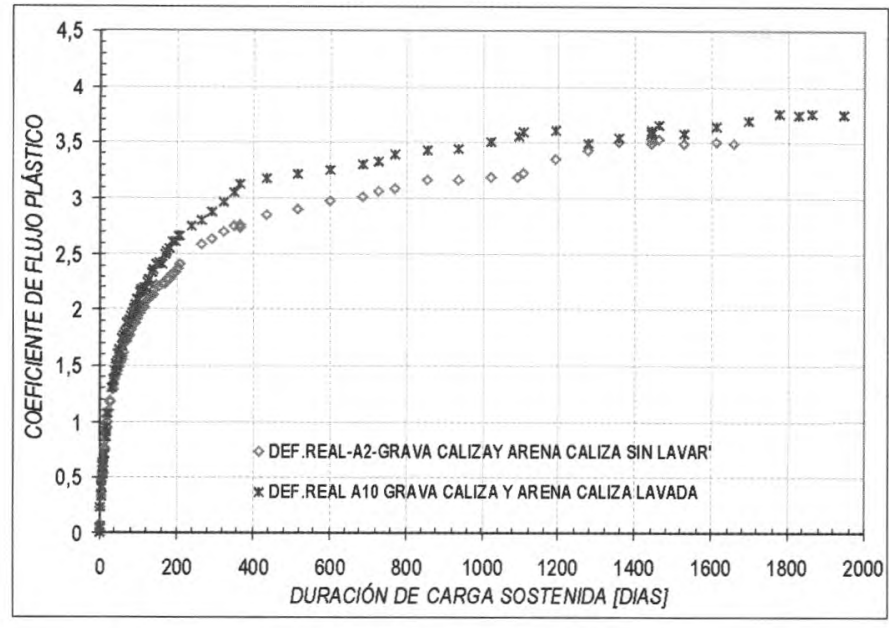

Figura 19. Comparativa de flujo plástico real de concreto con distintos agregados. Intervalo 1, 310<CC 326

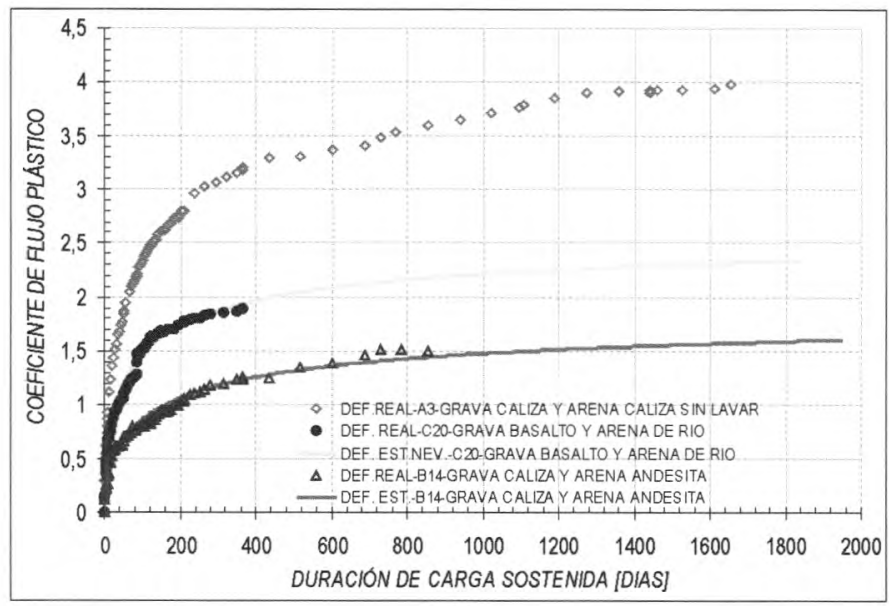

Figura 21. Comparativa de flujo plástico real para concreto con distintos agregados. Intervalo 3, 380 <CC $<401$

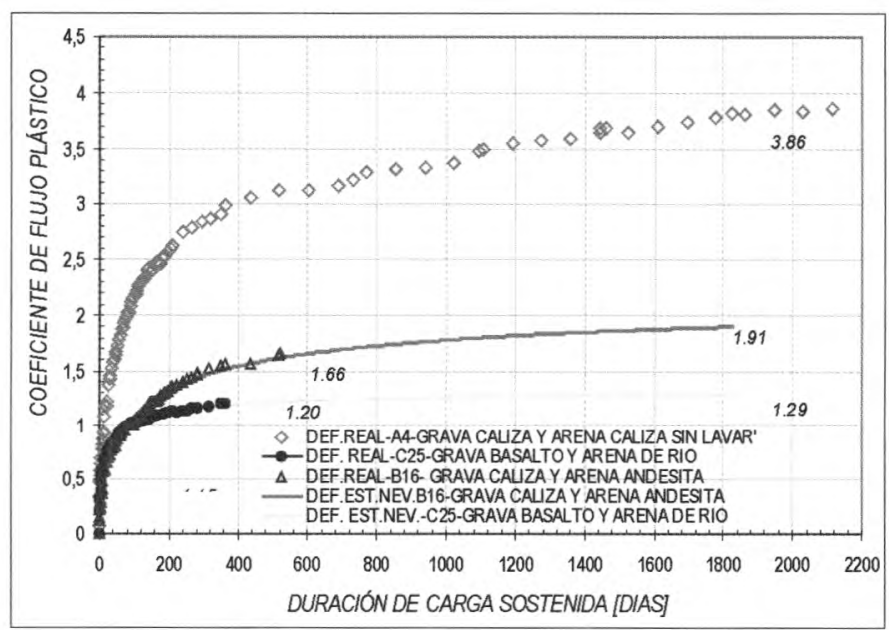

Figura 23. Comparativa de flujo plástico real de concreto con distintos agregados. Intervalo 5, 481 $<C C<505$

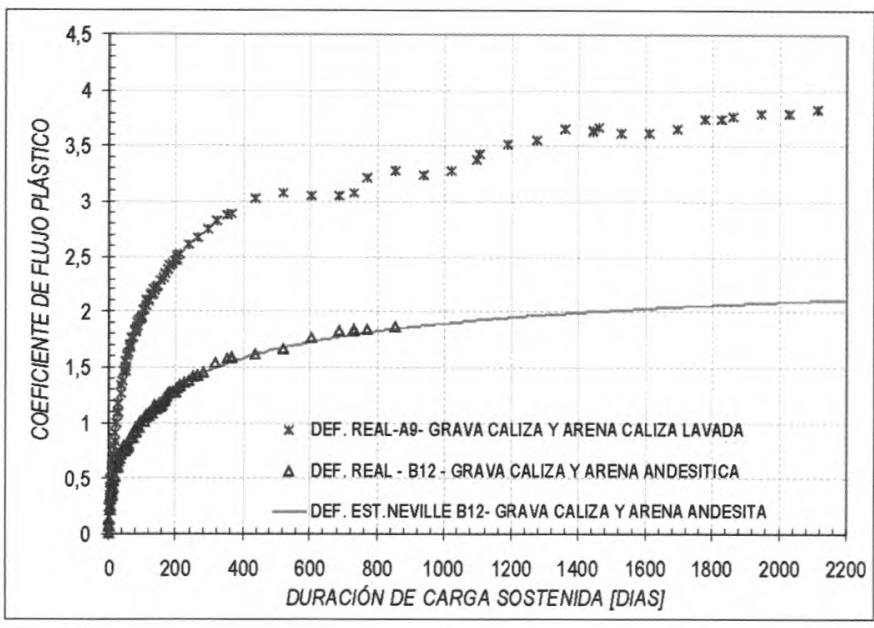

Figura 20. Comparativa de flujo plástico real de concreto con distintos agregados. Intervalo 2, $348<C C<366$

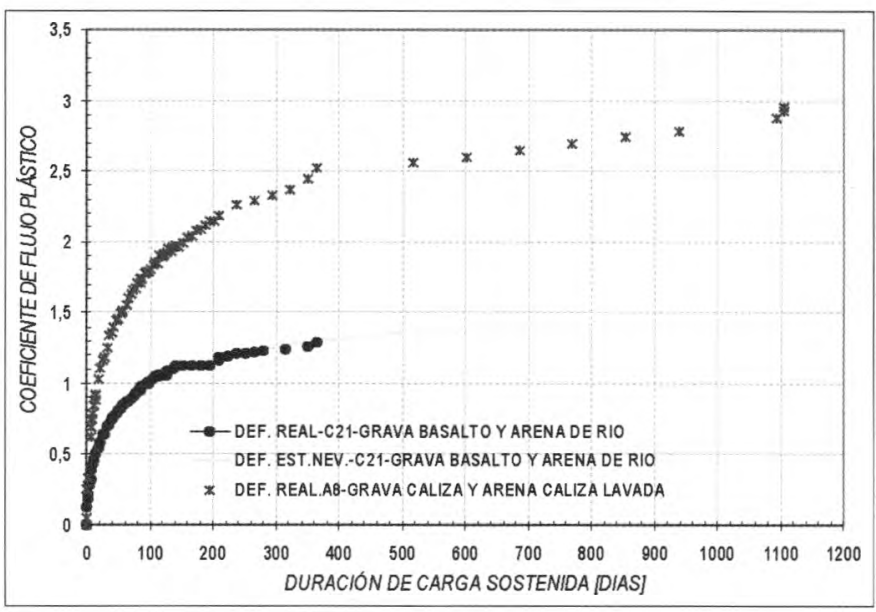

Figura 22. Comparativa de flujo plástico real de concreto con distintos agregados. Intervalo $4,440<C C<460$ 


\section{Semblanza de los autores}

Oscar Hernández-Castañeda. Obtuvo el título de ingeniero civil en la Facultad de Ingeniería, UNAM en el año 2001. Ha colaborado en Pemex Refinación y en la Dirección General de Carreteras Federales de la Secretaría de Comunicaciones y Transportes. Fue participante del programa Jóvenes hacia la Investigación organizado por la Coordinación de Investigación Científica de la UNAM, donde obtuvo el primer y tercer lugar de informe técnico a nivel licenciatura, en las estancias cortas de 1998 y 1999, respectivamente. Actualmente está realizando la maestría en ingeniería (Construcción) en la División de Estudios de Posgrado, Facultad de Ingeniería, UNAM. Es becario del CONACYT y del Instituto de Ingeniería, UNAM.

Alberto Leopoldo Fuentes-González. Es ingeniero civil por la Facultad de Ingeniería, UNAM desde 1977. Actualmente es investigador del Instituto de Ingeniería. Se ha dedicado al estudio de materiales para la industria de la construcción (concreto, ferrocemento, azufre) por más de 25 años. Ha participado en varios proyectos de investigación en el Instituto de Ingeniería para diferentes entidades y dependencias gubernamentales, así como para empresas privadas. Tiene varios artículos publicados y ha presentado una diversidad de ponencias y cursos relacionados con la ingeniería de materiales en México y en el extranjero. 